\title{
Efficacy and Safety of Sunitinib in Elderly Patients with Advanced Renal Cell Carcinoma at a University Hospital in Japan: Before Immuno-Oncology Therapy Era
}

\section{Tatsuya Takayama*, Akira Fujisaki, Satoshi Ando, Shinsuke Kurokawa and Tatsuo Morita}

Department of Urology, Jichi Medical University, Japan

\begin{abstract}
Introduction: The efficacy and safety of sunitinib in elderly patients with advanced renal cell carcinoma (RCC) remains poorly documented.

Methods: We assessed the efficacy and safety of sunitinib in first-line treatment of advanced RCC in the aged by reviewing the medical records of patients at Jichi Medical University Hospital, Japan. The patients were stratified into age $<75$ ( $\mathrm{n}=$ $38)$ and $\geq 75(n=9)$ years groups.

Results: Median progression-free survival in younger and older patients was comparable, at 7.3 vs. 6.7 months, respectively (HR, 1.101; 95\% CI: 0.399-3.039; $\mathrm{p}=0.8532$ ). Median overall survival was also comparable, at 19.2 vs. 11.8 months (HR, 0.775 ; $95 \%$ CI: $0.349-1.719 ; \mathrm{p}=0.5299$ ). Younger and older patients did not significantly differ in the frequency of any grade and grade 3 or more adverse events, or in the incidence of patients with grade 3 or more adverse events.
\end{abstract}

Conclusions: Treatment with sunitinib is effective and safe in elderly patients with advanced RCC although the limited sample size for older group can cause weak statistical power and only patients with no severe comorbidities were selected.

Keywords

Elderly, Renal cell carcinoma, Sunitinib, Efficacy, Safety

\section{Introduction}

Japan is a front-line country in the coming super-aging society. As with all cancers in the elderly [1], the frequency of renal cell carcinoma (RCC) is also increasing in Japan [2]. While clinical encounters with elderly patients with advanced RCC increase, these patients are only infrequently entered into clinical trials [3], and efficacy and safety data in this age range are scarce.

Sunitinib is an oral tyrosine-kinase inhibitor (TKI) of vascular endothelial receptor and other receptors which significantly prolongs the progression-free survival (PFS) [4-6] and overall survival (OS) $[5,6]$ of patients with metastatic RCC. Sunitinib also induces a range of adverse events, however, including symptomatic and laboratory toxicities; the former include hypertension, fatigue, diarrhea, nausea, vomiting, stomatitis, hand-foot syndrome and hypothyroidism while the latter include leukopenia, anemia, thrombocytopenia and increased creatinine [46]. Despite this apparently large number of treatment-related adverse events, prolonged treatment with sunitinib is not associated with new types or increased severity of treatment-related adverse events. Apart from hypothyroidism, toxicity is not cumulative [7]. In an expanded access trial of sunitinib, median PFS among patients aged over 65 years was 10.1 months compared to 8.9 months for the whole population. Except for fatigue, patients aged over 65 years appear to have experienced no worse grade 3-4 non-hematological adverse events than the whole population of patients [5].

In general, while people aged over 65 years are con-

*Corresponding author: Tatsuya Takayama, Department of Urology, Jichi Medical University, 3311-1 Yakushiji Shimotuske, Tochigi 329-0498, Japan, Tel: +81-285-587379, Fax: +81-285-40-6595, E-mail: ttakayam@jichi.ac.jp

Received: March 13, 2018; Accepted: April 14, 2018; Published online: April 16, 2018

Citation: Takayama T, Fujisaki A, Ando S, et al. (2018) Efficacy and Safety of Sunitinib in Elderly Patients with Advanced Renal Cell Carcinoma at a University Hospital in Japan: Before Immuno-Oncology Therapy Era. J Ren Cancer 1(1):1-6

Copyright: (C) 2018 Takayama T, et al. This is an open-access article distributed under the terms of the Creative Commons Attribution License, which permits unrestricted use, distribution, and reproduction in any medium, provided the original author and source are credited. 
Citation: Takayama T, Fujisaki A, Ando S, et al. (2018) Efficacy and Safety of Sunitinib in Elderly Patients with Advanced Renal Cell Carcinoma at a University Hospital in Japan: Before Immuno-Oncology Therapy Era. J Ren Cancer 1(1):1-6

sidered elderly, age-related physiological changes occur predominantly in those between 70 and 75 years. Physiological function decreases as age progresses beyond 75 years $[8,9]$, yet few papers define a cut-off point for older cancer patients of 75 years. Our present study is the first to evaluate the efficacy and safety of first-line anticancer

Table 1: Patient characteristics classified by age

\begin{tabular}{|c|c|c|c|}
\hline Variable & $\begin{array}{c}\text { Age }<75 \text { years } \\
(n=38)\end{array}$ & Age $\geq 75$ years $(n=9)$ & $p$ values \\
\hline Median (range) age, years & $62(37-74)$ & $77(75-84)$ & $<0.0001$ \\
\hline Male/female, \% & $76 / 24$ & $90 / 10$ & 0.4073 \\
\hline Hypertension, $n(\%)$ & $20(53)$ & $6(67)$ & 0.4463 \\
\hline Diabetes mellitus, $\mathrm{n}(\%)$ & $8(22)$ & $2(22)$ & 0.9386 \\
\hline Smoking history, $\mathrm{n}(\%)$ & $21(55)$ & $5(56)$ & 0.8467 \\
\hline Prior nephrectomy, n(\%) & $15(39)$ & $4(44)$ & 0.7847 \\
\hline \multicolumn{4}{|l|}{ ECOG PS, $n(\%)$} \\
\hline 0 & $21(55)$ & $3(33)$ & \multirow{3}{*}{0.2073} \\
\hline 1 & $9(24)$ & $5(56)$ & \\
\hline$\geq 2$ & $6(16)$ & $1(11)$ & \\
\hline Missing (unknown) & $2(5)$ & $0(0)$ & \\
\hline \multicolumn{4}{|l|}{ Risk factors based on MSKCC data, $n(\%)$} \\
\hline 0 (favorable) & $4(11)$ & $0(0)$ & \multirow{4}{*}{0.2161} \\
\hline 1-2 (intermediate) & $21(55)$ & $8(89)$ & \\
\hline$\geq 3$ (poor) & $11(29)$ & $1(11)$ & \\
\hline missing & $2(5)$ & $0(0)$ & \\
\hline \multicolumn{4}{|l|}{ Common site of metastasis, $\mathrm{n}(\%)$} \\
\hline Lung & $21(55)$ & $6(67)$ & 0.5338 \\
\hline Bone & $11(29)$ & $3(33)$ & 0.7959 \\
\hline Lymph nodes & $10(26)$ & $3(33)$ & 0.6722 \\
\hline \multicolumn{4}{|l|}{ Histology, $\mathrm{n}(\%)$} \\
\hline Clear cell & $19(50)$ & $6(67)$ & \multirow{3}{*}{0.3384} \\
\hline Others & $3(8)$ & $0(0)$ & \\
\hline Missing (untraced or unknown) & $16(42)$ & $3(33)$ & \\
\hline \multicolumn{4}{|l|}{ Initial doses of sunitinib, $\mathrm{n}(\%)$} \\
\hline \multicolumn{4}{|l|}{$50 \mathrm{mg}$} \\
\hline 4 weeks on, 2 weeks off & $27(70)$ & $5(56)$ & \\
\hline 2 weeks on, 1 weeks off & $3(8)$ & $0(0)$ & \\
\hline \multicolumn{4}{|l|}{$37.5 \mathrm{mg}$} \\
\hline 4 weeks on, 2 weeks off & $6(16)$ & $3(33)$ & \multirow[t]{2}{*}{$0.1483^{*}$} \\
\hline 2 weeks on, 1 weeks off & $0(0)$ & 1(11) & \\
\hline \multicolumn{4}{|l|}{$25 \mathrm{mg}$} \\
\hline 4 weeks on, 2 weeks off & 1(3) & $0(0)$ & \\
\hline 2 weeks on, 1 weeks off & 1(3) & $0(0)$ & \\
\hline \multicolumn{4}{|l|}{ Relative dose intensity, \% } \\
\hline median & 75.0 & 68.8 & 0.7559 \\
\hline mean $\pm S D$ & $72.5 \pm 19.4$ & $70.4 \pm 15.9$ & 0.8105 \\
\hline \multicolumn{4}{|l|}{ Clinical outcome of sunitinib treatment, $n(\%)$} \\
\hline Continuing sunitinib & $3(8)$ & $0(0)$ & \multirow{2}{*}{0.3837} \\
\hline Discontinuing sunitinib & $35(92)$ & $9(100)$ & \\
\hline \multicolumn{4}{|l|}{ Reason for discontinuation $(n=42)$} \\
\hline Lack of efficacy & $17(44)$ & $6(67)$ & \\
\hline Progressive disease & $16(41)$ & $5(56)$ & \\
\hline Stable disease with increasing tendency & 1(3) & $0(0)$ & \\
\hline Death & $0(0)$ & $1(11)$ & 0.5019 \\
\hline Adverse events & $15(40)$ & $3(33)$ & \\
\hline Others & $3(8)$ & $0(0)$ & \\
\hline
\end{tabular}

All statistical analysis was carried out except for missing data. Mean and median relative dose intensity (\%) was calculated during the first 6 weeks of treatment. * $50 \mathrm{mg}$ vs $\leq 37.5 \mathrm{mg} ; \mathrm{p}=0.1787$

Abbreviations: ECOG PS, Eastern Cooperative Oncology Group performance status; MSKCC, Memorial Sloan-Kettering Cancer Center; SD, standard deviation 
Citation: Takayama T, Fujisaki A, Ando S, et al. (2018) Efficacy and Safety of Sunitinib in Elderly Patients with Advanced Renal Cell Carcinoma at a University Hospital in Japan: Before Immuno-Oncology Therapy Era. J Ren Cancer 1(1):1-6

treatment with sunitinib stratified by age 75 years.

\section{Materials and Methods}

The study was conducted as a retrospective analysis of 47 patients with advanced RCC treated with sunitinib in the first-line setting, except for use in neoadjuvant or presurgical therapy, between May 2008 and August 2016 at the Department of Urology, Jichi Medical University Hospital. The observation period for each patient extended from the start of sunitinib treatment until disease progression, death or August 2016, whichever occurred first.

Data for age, sex, hypertension, diabetes mellitus, smoking history, prior nephrectomy, Eastern Cooperative Oncology Group performance status, Memorial Sloan-Kettering Cancer Center data, common site of metastasis, histology, treatment dose and schedule of sunitinib, relative dose intensity, PFS, OS and adverse events were obtained from medical records. Protocols were approved by the Institutional Research Review Boards of Jichi Medical University with an opt-out system (No. A14-129). Toxic effects were registered according to Common Terminology Criteria for Adverse Events version 3.0 (CTCAE v3.0). Response was assessed by the treating physician according to the Response Evaluation Criteria in Solid Tumor (RECIST) 1.0 criteria.

Patients were divided into age groups of $<75$ and $\geq 75$ years. Categorical variables were expressed as frequencies and percentages, and differences were compared using Pearson's chi-square test. PFS and OS curves were depicted using the Kaplan-Meier method and compared with the log-rank test. All $\mathrm{P}$ values presented are two-sid-

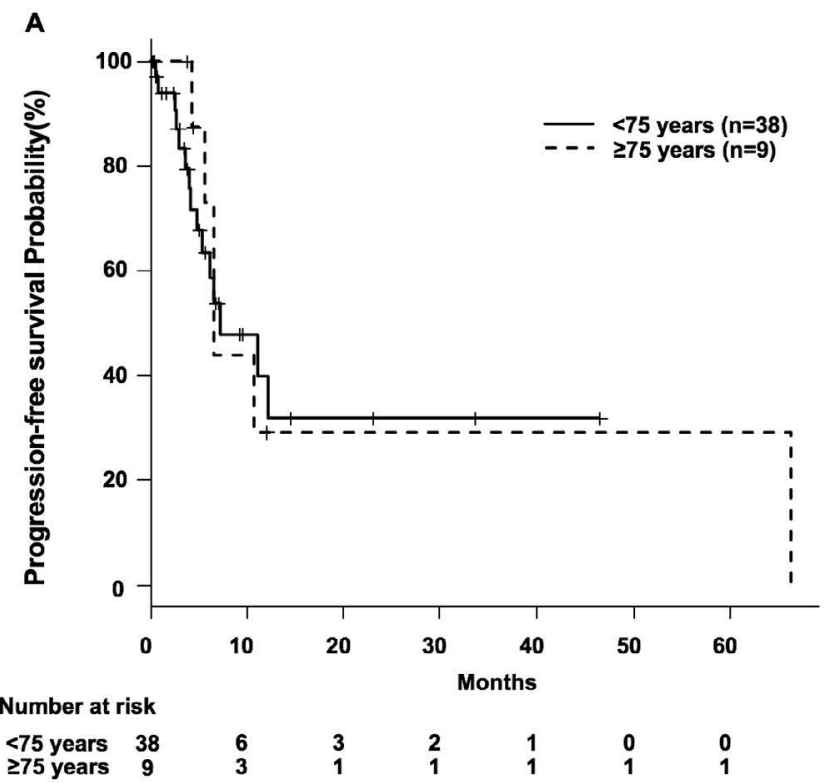

ed. Statistical significance was calculated using StatView ver. 5 (Abacus Concepts, CA, USA), with $\mathrm{P}$ values of $<$ 0.05 considered to indicate statistical significance.

\section{Results}

The study included 47 patients with advanced RCC who were treated with sunitinib as first-line treatment, except for use in neoadjuvant or presurgical therapy. There were 38 and 9 patients aged $<75$ and $\geq 75$ years, respectively. Baseline characteristics of the patients and data on treatment exposure to sunitinib are shown in Table 1. Baseline characteristics between the two groups were similar. Among the two groups, $71 \%$ of patients aged $<75$ were administered sunitinib at $50 \mathrm{mg} /$ day orally in a 4 weeks on, 2 weeks off regimen, as were $56 \%$ of those aged $\geq 75$ years. In contrast, starting dose of sunitinib did not significantly differ between the two age groups even if $50 \mathrm{mg}$ vs. $\leq 37.5 \mathrm{mg}$ daily. Further, mean and median relative dose intensity during the first 6 weeks of treatment was similar between the two groups. The median follow-up was 16.9 months (range, 0.6-80.9 months). Three patients aged $<75$ continued sunitinib treatment. The most common reasons for discontinuation were progressive disease and adverse events, with no significant difference in reasons between the two groups. Other reasons for discontinuation included complete response $(\mathrm{n}=1)$, cost concerns $(\mathrm{n}=1)$ and patient refusal $(\mathrm{n}=1)$. Twenty-two patients $(58 \%)$ aged $<75$ and 6 patients (66\%) aged $\geq 75$ years received second-line treatment. The former consisted of axitinib $(\mathrm{n}=8)$, everolimus $(n=7)$, sorafenib $(n=4)$, temsirolimus $(n=2)$ and

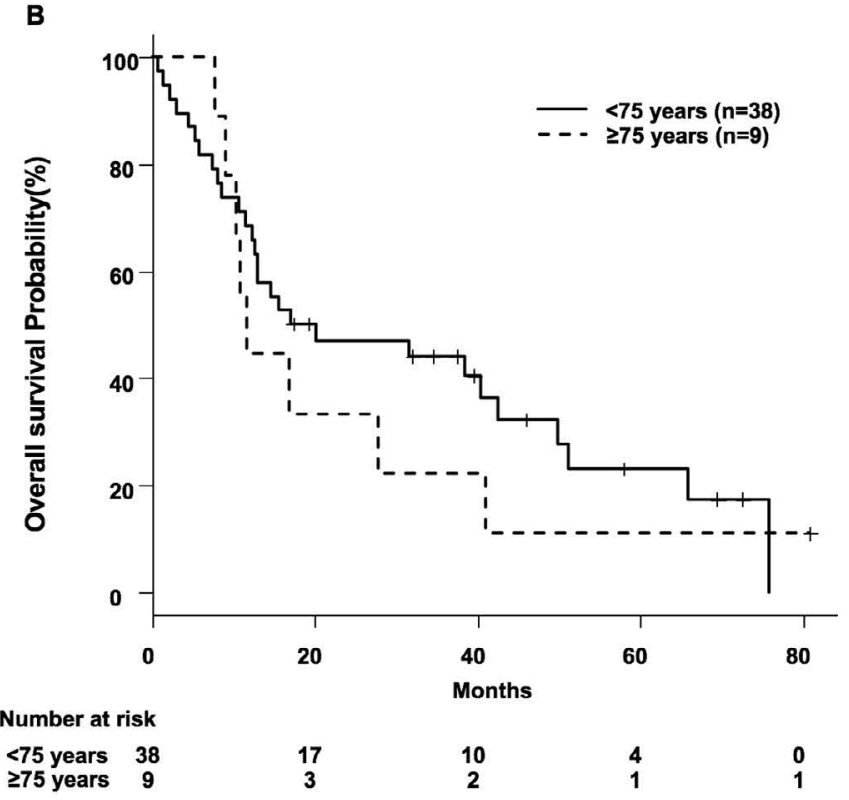

Figure 1: Kaplan-Meier curves of progression-free survival $(A)$ and overall survival $(B)$ in sunitinib-treated patients by age $(<$ 75 vs. $\geq 75$ years).

There were no significant differences in PFS or OS between the $<75$ and $\geq 75$-year groups. 
Citation: Takayama T, Fujisaki A, Ando S, et al. (2018) Efficacy and Safety of Sunitinib in Elderly Patients with Advanced Renal Cell Carcinoma at a University Hospital in Japan: Before Immuno-Oncology Therapy Era. J Ren Cancer 1(1):1-6

cytokine ( $\mathrm{n}=1)$ and the latter consisted of axitinib ( $\mathrm{n}$ $=2)$, everolimus $(n=3)$ and sorafenib $(n=1)$. The next treatments did not incorporate anti-programmed death 1 therapy during the observation period.

We compared the difference in PFS and OS in the two groups. Median PFS in the younger and older patients was comparable, at 7.3 vs. 6.7 months, respectively (HR, 1.101; 95\% CI: 0.399-3.039; $\mathrm{p}=0.8532$ ), as was median OS, at 19.2 vs. 11.8 months (HR, 0.775; 95\% CI: 0.349 $1.719 ; \mathrm{p}=0.5299$ ), with neither of these differences being significant (Figure 1). The best response of sunitinib treatment in patients aged $<75$ was composed of CR ( $\mathrm{n}$ $=1), P R(n=1), S D(n=30)$ and PD $(n=6)$, as was SD $(n=9)$ in those aged $\geq 75$ years.
Table 2 shows adverse events occurring in at least $20 \%$ of patients or at grade 3 or 4 . Symptomatic adverse events with a frequency of more than $30 \%$ were nausea, fever, fatigue, anorexia, hand-foot syndrome, hypertension, and stomatitis. There was no significant difference in adverse events of any grade or of grade 3 between the two groups, except for a significant difference in the incidence of grade 3 diarrhea, albeit that this occurred in only one patient aged $\geq 75$ years. Abnormal laboratory data with a frequency of more than $40 \%$ were AST increased, ALP increased, platelet count decreased, ALT increased, hypothyroidism, creatinine increased, neutrophil count decreased, leukocyte count decreased, hyponatremia, blood urea nitrogen increased and hypocalcemia. These showed no significant difference for any grade

Table 2: Adverse events in sunitinib-treated patients classified by age

\begin{tabular}{|c|c|c|c|c|c|c|}
\hline \multirow{3}{*}{ Adverse Event } & \multicolumn{4}{|c|}{ Variable } & \multirow{2}{*}{\multicolumn{2}{|c|}{$p$ values }} \\
\hline & \multicolumn{2}{|c|}{ Age $<75$ years $(n=38)$} & \multicolumn{2}{|c|}{ Age $\geq 75$ years $\quad(n=9)$} & & \\
\hline & All grade & $\geq$ Grade 3 & All grade & $\geq$ Grade 3 & All grade & $\geq$ Grade 3 \\
\hline \multicolumn{7}{|l|}{ Symptoms, n(\%) } \\
\hline Nausea & $20(53)$ & $0(0)$ & $3(33)$ & $0(0)$ & 0.2977 & - \\
\hline Fever & $16(42)$ & $0(0)$ & $3(33)$ & $0(0)$ & 0.6297 & - \\
\hline Fatigue & 15(39) & $0(0)$ & $3(33)$ & $0(0)$ & 0.7333 & - \\
\hline Anorexia & $15(39)$ & $2(5)$ & $3(33)$ & $2(22)$ & 0.7333 & 0.1011 \\
\hline Hand-foot syndrome & $14(37)$ & $3(8)$ & $4(44)$ & $2(22)$ & 0.6731 & 0.216 \\
\hline Hypertension (de novo) & $14(37)$ & $2(5)$ & $4(44)$ & $0(0)$ & 0.6731 & 0.4818 \\
\hline Stomatitis & $13(34)$ & $2(5)$ & $3(33)$ & $1(11)$ & 0.9602 & 0.5187 \\
\hline Diarrhea & $10(26)$ & $0(0)$ & $3(33)$ & $1(11)$ & 0.6722 & 0.0378 \\
\hline Peripheral edema & $6(16)$ & $0(0)$ & $2(22)$ & $0(0)$ & 0.6443 & - \\
\hline Gastrointestinal hemorrhage & $5(13)$ & $1(3)$ & $2(22)$ & $0(0)$ & 0.4922 & 0.6228 \\
\hline \multicolumn{7}{|l|}{ Abnormal laboratory data, $n(\%)$} \\
\hline AST increased & $31(82)$ & $2(5)$ & $8(89)$ & $0(0)$ & 0.5998 & 0.4818 \\
\hline ALP increased & $25(66)$ & $1(3)$ & $6(67)$ & $0(0)$ & 0.9602 & 0.6228 \\
\hline Platelet count decreased & $24(63)$ & $14(37)$ & $7(78)$ & $3(33)$ & 0.4053 & 0.8438 \\
\hline ALT increased & $22(58)$ & $3(8)$ & $6(67)$ & $0(0)$ & 0.6297 & 0.3837 \\
\hline Hypothyroidism* & $21(55)$ & $2(5)$ & $2(22)$ & $0(0)$ & 0.0746 & 0.4818 \\
\hline Creatinine increased & $20(53)$ & $1(3)$ & $4(44)$ & $0(0)$ & 0.8349 & 0.6382 \\
\hline Neutrophil count decreased & $19(50)$ & $10(26)$ & $6(67)$ & $3(33)$ & 0.3676 & 0.6722 \\
\hline Leukocyte count decreased & $17(45)$ & $11(29)$ & $6(67)$ & $3(33)$ & 0.2367 & 0.7959 \\
\hline Hyponatremia & $17(45)$ & $4(11)$ & $4(44)$ & $0(0)$ & 0.9354 & 0.3019 \\
\hline Blood urea nitrogen increased & $16(42)$ & $1(3)$ & $5(56)$ & $0(0)$ & 0.506 & 0.618 \\
\hline Hypocalcemia & 15(39) & $1(3)$ & $4(44)$ & $0(0)$ & 0.7847 & 0.6228 \\
\hline Hypophosphatemia & $14(37)$ & $4(11)$ & $2(22)$ & $0(0)$ & 0.4053 & 0.3089 \\
\hline Hyperkalemia & 13(34) & $0(0)$ & $3(33)$ & $0(0)$ & 0.9602 & - \\
\hline Gamma-GTP increased & $12(32)$ & $2(5)$ & $2(22)$ & $0(0)$ & 0.581 & 0.4818 \\
\hline Serum amylase increased & $12(32)$ & $3(8)$ & $2(22)$ & $0(0)$ & 0.581 & 0.3837 \\
\hline Hyperuricemia & $12(32)$ & $0(0)$ & $2(22)$ & $0(0)$ & 0.7132 & - \\
\hline Hypercalcemia & $9(24)$ & $0(0)$ & $1(11)$ & $0(0)$ & 0.4073 & - \\
\hline Hypokalemia & $8(21)$ & $1(3)$ & $1(11)$ & $0(0)$ & 0.4955 & 0.6228 \\
\hline lipase Increased & $6(16)$ & $3(8)$ & $2(22)$ & $1(11)$ & 0.6443 & 0.7559 \\
\hline Total bilirubin increased & $6(16)$ & $0(0)$ & $2(22)$ & $0(0)$ & 0.6443 & - \\
\hline
\end{tabular}

Graded according to CTCAEv3.0, and occurring in at least $20 \%$ of patients, or including patients with grade 3 or 4 adverse events.

*: Hypothyroidism consists of thyroid hormone decreased and thyroid-stimulating hormone increased, and most patients received medication before symptom appearance.

Abbreviations: AST: aspartate aminotransferase; ALP: alkaline phosphatase; ALT: alanine aminotransferase; GTP: glutamyl transpeptidase 
Citation: Takayama T, Fujisaki A, Ando S, et al. (2018) Efficacy and Safety of Sunitinib in Elderly Patients with Advanced Renal Cell Carcinoma at a University Hospital in Japan: Before Immuno-Oncology Therapy Era. J Ren Cancer 1(1):1-6

Table 3: Incidence of $\geq$ Grade 3 adverse event in sunitinib-treated patients classified by age

\begin{tabular}{|c|c|c|c|c|c|}
\hline \multirow{3}{*}{ Contents } & \multicolumn{4}{|c|}{ Variable } & \multirow{3}{*}{$P$ value } \\
\hline & \multicolumn{2}{|c|}{ Age $<75$ years $(n=38)$} & \multicolumn{2}{|c|}{ Age $\geq 75$ years $(n=9)$} & \\
\hline & + & - & + & - & \\
\hline$\geq$ Grade 3 in all Adverse Events & $30(79)$ & $8(21)$ & $9(100)$ & $0(0)$ & 0.1308 \\
\hline$\geq$ Grade 3 in Symptoms & $9(24)$ & $29(76)$ & $5(56)$ & $4(44)$ & 0.0601 \\
\hline$\geq$ Grade 3 in Abnormal laboratory data & $25(66)$ & $13(34)$ & $5(56)$ & $4(44)$ & 0.5656 \\
\hline
\end{tabular}

or for grade 3 between the two groups. Seven patients experienced grade 4 adverse events (data not shown), of whom six were $<75$ years. These included platelet count decreased $(n=5)$, neutrophil count decreased $(n=1)$, leukocyte count decreased $(n=1)$, lipase increased ( $n$ $=2$ ) and hypophosphatemia $(n=1)$; of these, the three former adverse events occurred in one patient aged $<75$ years. Table 3 reports the incidence grade 3 or more adverse events, showing that there was no significant difference in the incidence of any grade 3 adverse event even on classification into symptomatic and abnormal laboratory events.

\section{Discussion}

The main finding of this study is that treatment with sunitinib was effective and safe in elderly patients with advanced RCC in a university hospital in Japan. Median PFS, OS and frequencies and incidences of adverse events in patients aged $<75$ and $\geq 75$ years were comparable although the limited sample size for older group can cause weak statistical power.

Sunitinib, an oral tyrosine-kinase inhibitor (TKI) of vascular endothelial receptor and other receptors, is recommended in various guidelines as first-line treatment of metastatic or advanced RCC. In an expanded access trial, $32 \%$ of 4371 patients who received sunitinib were 65 years or older. Median PFS, OS and the frequencies of the most common grade 3-4 treatment-related adverse events in the elderly subgroup were comparable to those in the entire study population [10]. Using pooled data from six prospective trials, efficacy and toxicity of sunitinib was compared in metastatic RCC patients categorized by age 70 years [11]. Results showed that median PFS and OS were comparable between the groups. While older patients suffered from more fatigue, cough, peripheral edema, anemia, decreased appetite, weight decrease and thrombocytopenia, with a frequency of $20 \%$ or more, and also had more grade 3 toxicity, patients younger than 70 years more often experienced hand-foot syndrome and hair color changes, with a frequency of $20 \%$ or more. In contrast, there was no difference between the groups in grade 4 toxicity or treatment-related deaths. In a retrospective registry-based study in the Czech Republic, 1315 patients treated with sunitinib as first-line therapy were divided into those aged $<70(\mathrm{n}=1016)$ and $\geq 70$ years $(n=299)$ and analyzed for the safety and activi- ty of sunitinib. Median OS was extended in the younger population while discontinuation rate was higher in the elderly population [12]. In contrast, a retrospective study of 327 patients with advanced RCC in two Chinese clinical centers who were treated with sunitinib or sorafenib stratified the patients into three groups, namely young (aged < 45 years), middle-aged (aged 45-64) and old (aged $\geq 65$ years). Old age was an independent favorable prognostic factor for OS and PFS compared with younger age, whereas the frequencies of adverse events among the three groups were similar [13]. A retrospective study based on data from a comprehensive geriatric assessment (CGA) for sunitinib safety and activity in 68 patients aged $\geq 70$ years treated with sunitinib and analyzed for frailty showed that although sunitinib was effective, early interruption was frequent [14]. Rates of adverse events in that study were higher than those in the expanded access trial [10] and pooled data analysis [11] mentioned above. No correlation was found between frailty at CGA and toxicity or treatment response. To our knowledge, only one other study has stratified patients by age 75 years, the International Metastatic RCC Database Consortium study. That study enrolled 1381 patients with metastatic RCC and determined the efficacy but not safety of targeted therapy which included sunitinib, sorafenib, bevacizumab and AZD217. The distribution of patients by age, histology, performance status and risk criteria was closely similar to that in the present study, but the frequency of prior nephrectomy was $70 \%$ or more versus our $40 \%$. Age $\geq 75$ years was not found to be associated with poor OS [15].

Taken together, these results indicate a tendency toward acceptable efficacy of sunitinib treatment in elderly patients with advanced or metastatic RCC, whereas safety remains controversial. Moreover, our present study is the only one to have examined both the efficacy and safety of sunitinib treatment in the first-line setting in patients with advanced RCC divided by age 75 years. Given the well-known difference between 'chronological' versus 'biological' age, it is particularly difficult to clarify the best cut-off point dividing 'young' and 'old'. Given the sharp increase in age-related physiological changes between 70 and 75 years, however, the cut-off of 75 years appears to better reflect the complexity of cancer, comorbidities, and old age [8,9]. Nevertheless, the cut-off point should be considered with particular care in countries with a long life expectancy, such as Japan [16,17]. 
Citation: Takayama T, Fujisaki A, Ando S, et al. (2018) Efficacy and Safety of Sunitinib in Elderly Patients with Advanced Renal Cell Carcinoma at a University Hospital in Japan: Before Immuno-Oncology Therapy Era. J Ren Cancer 1(1):1-6

In the present study, the efficacy of sunitinib treatment was similar between the two age groups, although mean PFS and OS were somewhat shorter than in these previous reports due to the lower frequency of prior nephrectomy and low incidence of patients with a favorable MSKCC risk profile. Moreover, among reasons for moving to next treatment, progressive disease and adverse events also occurred with increasing frequency in a post-marketing study which collected sunitinib safety and efficacy data in 1689 Japanese patients with advanced RCC [18]. Among adverse events, the frequency of increased liver enzymes in our present series was higher than in this previous study while the frequency of grade 3 or more was less $10 \%$. The incidence of grade or grade 3 or more adverse events was compared in those aged $<75$ and $\geq 75$ years under almost the same relative dose intensity without variation in starting dose by age (data not shown). In addition, the incidence of comorbidities such as hypertension, diabetes mellitus and smoking history was similar between the two groups and did not differ to that in the previous reports. Seven patients had Grade 4 adverse events; among these, six were aged $<75$ years, while the patient aged $\geq 75$ years experienced a non-symptomatic increase in lipase followed by improvement when sunitinib dose was decreased from $50 \mathrm{mg}$ to $37.5 \mathrm{mg}$. This allowed the continuation of sunitinib until the development of progressive disease. We therefore assume that adverse events depend not only on age but also on individual characteristics or ethnicity.

Limitations of the present study include its small sample size and the bias inherent to retrospective studies. In addition, our study did not include patients with severe comorbidities and did not assure the appropriateness of stratification by age 75 -years. Given the well-known difficulty in enrolling elderly patients in prospective clinical trials because of comorbidities and concerns over the toxic effects of treatment, a large scale retrospective study with high quality is warranted. Nevertheless, the present study provides additional data on the relationship between age and sunitinib treatment in patients with advanced RCC treated in a Japanese university hospital.

\section{Conflict of interest}

The authors declare that they have no conflict of interest.

\section{References}

1. SEER Cancer Statistics Review (CSR) 1975-2014.

2. Kanayama HO, Fukumori T, Fujimoto H, et al. (2015) The first large-scale multicenter study from the Cancer Registration Committee of the Japanese Urological Association. Int J Urol 22: S1-S7.
3. Aapro MS, Köhne $\mathrm{CH}$, Cohen HJ, et al. (2005) Never too old? Age should not be a barrier to enrollment in cancer clinical trials. Oncologist 10: 198-204.

4. Motzer RJ, Hutson TE, Tomczak P, et al. (2007) Sunitinib versus interferon alfa in metastatic renal-cell carcinoma. $\mathrm{N}$ Engl J Med 356: 115-124.

5. Motzer RJ, Hutson TE, Tomczak P, et al. (2009) Overall survival and updated results for sunitinib compared with interferon alfa in patients with metastatic renal cell carcinoma. J Clin Oncol 27: 3584-3590.

6. Gore ME, Szczylik C, Porta C, et al. (2015) Final results from the large sunitinib global expanded-access trial in metastatic renal cell carcinoma. $\mathrm{Br} \mathrm{J}$ Cancer 113: 12-19.

7. Porta C, Gore ME, Rini BI, et al. (2016) Long-term safety of sunitinib in metastatic renal cell carcinoma. Eur Urol 69: 345-351.

8. Surbone A, Kagawa Singer M, Terret C, et al. (2007) The illness trajectory of elderly cancer patients across cultures: SIOG position paper. Ann Oncol 18: 633-638.

9. Given B, Given C, Azzouz F, et al. (2001) Physical functioning of elderly cancer patients prior to diagnosis and following initial treatment. Nurs Res 50: 222-232.

10. Gore ME, Szczylik C, Porta C, et al. (2009) Safety and efficacy of sunitinib for metastatic renal-cell carcinoma: An expanded-access trial. Lancet Oncol 10: 757-763.

11. Hutson TE, Bukowski RM, Rini BI, et al. (2014) Efficacy and safety of sunitinib in elderly patients with metastatic renal cell carcinoma. Br J Cancer 110: 1125-1132.

12. Poprach A, Lakomy R, Bortlicek Z, et al. (2014) Efficacy of Sunitinib in Elderly Patients with Metastatic Renal Cell Carcinoma: Data from Real-World Clinical Practice. Drugs Aging 33: 655-663.

13. Zhang G, Zhu Y, Dong D, et al. (2014) Clinical outcome of advanced and metastatic renal cell carcinoma treated with targeted therapy: Is there a difference between young and old patients? Onco Targets Ther 7: 2043-2052.

14. Brunello A, Basso U, Sacco C, et al. (2013) Safety and activity of sunitinib in elderly patients ( $\geq 70$ years) with metastatic renal cell carcinoma: A multicenter study. Ann Oncol 24: 336-342.

15. Khambati HK, Choueiri TK, Kollmannsberger CK, et al. (2014) Efficacy of targeted therapy for metastatic renal cell carcinoma in the elderly patient population. Clin Genitourin Cancer 12: 354-358.

16. Naito S, Tomita Y, Rha SY, et al. (2014) Kidney Cancer Working Group report. Jpn J Clin Oncol 40: 51-56.

17. Stafford HS, Saltzstein SL, Shimasaki S, et al. (2008) Racial/ethnic and gender disparities in renal cell carcinoma incidence and survival. J Urol 179: 1704-1708.

18. Akaza H, Naito S, Ueno N, et al. (2015) Real-world use of sunitinib in Japanese patients with advanced renal cell carcinoma: efficacy, safety and biomarker analyses in 1689 consecutive patients. Jpn J Clin Oncol 45: 576-583. 